\title{
MODELO PARA EL ESTUDIO DEL CAMPO DE LA PERCEPCION VISUAL EN LOS ARBITROS PROFESIONALES DE NORTE DE SANTANDER
}

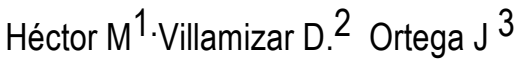 \\ 1. Estudiante de Maestría CAFD Licenciado en Educación Física Recreación y Deportes. \\ Universidad de Pamplona. sportmendoza2010@hotmail.com
}

2. Doctora en Salud: Envejecimiento y Cognición: Una Perspectiva Psicológica Universidad Rovira i Virgili España. Psicóloga. Especialista en Orientación Educativa, Familiar y Comunitaria. Docente Asociada Programa de Psicología. Universidad de Pamplona. jacarrillo71@hotmail.com

3. Doctor en Ciencias de la Cultura Física. Docente programa Educación Física Recreación y deportes. Universidad de Pamplona arlesjavierortegap@hotmail.com

\section{RESUMEN}

El modelo para el estudio del campo de la percepción visual en los árbitros profesionales del futbol del Norte de Santander surge como una necesidad científica en la tare a de arbitrar el futbol, si nos damos cuenta en este tema son muy pocas las investigaciones, se realizó este trabajo de investigación dirigida por una secuencia progresiva en el desarrollo del campo de la percepción visual, se tuvieron en cuenta tres fases distribuidas de la siguiente manera : fase uno denominada fase de diagnóstico en donde se aplicó examen de agudeza visual a los colegiados objeto de estudio para saber de su condición en esta parte de la visión, se utilizó el examen de campimetría para conocer el estado de la visión de los colegiados en este estadio de la visión, se manejó aplicación de los estilos de personalidad MIPS en la muestra de estudio, se empleó el WAIS IV escala de inteligencia, con el fin de detectar la relación del campo perceptivo con las variables psicologías del arbitraje, en la segunda fase denominada fase de preparación se aplicaron actividades que desarrollan las habilidades visuales que comprometen el manejo del campo de la percepción visual, en la fase tres: fase de situación real se desarrollaron cuatro partidos de futbol en donde se hizo un seguimiento de rendimiento arbitral evaluando su desempeño, en donde se comprobó que la intervención influyo positivamente en el desarrollo arbitral al detectar un rendimiento de alto porcentaje de aciertos. Se creó un modelo para el estudio del campo de la percepción visual, se detectaron las relaciones de las variables psicológicas con el campo perceptivo visual en la fase de diagnóstico, fase de preparación y en la fase de situación real.

Palabras claves: percepción visual, arbitraje, campo visual, variables psicológicas.

\begin{abstract}
The model for the study of the field of visual perception in professional soccer referees of Norte de Santander emerges as a scientific necessity in the task of refereeing football if we realize, there are few investigations about this topic, this research was conducted by a progressive sequence in the development of the field of visual perception, there were taken into account three phases distributed as follows: Phase One: it is called diagnostic phase where it was applied a visual acuity exam to the students object of study to know their condition in this part of the vision, it was used the visual field examination to view the status of students in this stadium vision, it was handled implementation of MIPS personality styles in the study sample, it was used the IV WAIS intelligence scale, in order to detect the relationship of the perceptual field with the varying psychologies of arbitration. In phase two called preparation phase were applied activities that develop the visual skills that involve the management of the field of visual perception. In phase three, real situation phase, four football matches were developed where it was carried out a monitoring arbitration performance to evaluate their efficiency, where it was found that
\end{abstract}


the intervention was positively influenced in the arbitration development to detect a performance with high successful percentage. It was created a model for the study of the field of visual perception, there were detected the relationships of psychological variables with visual perceptual field in the diagnostic phase, preparation phase and real situation phase.

Keywords: visual perception, arbitration, visual field, psychological variables.

\section{Convocatoria III. Trimestre. Recibido el 15 de junio de 2017; Aceptado el 15 de julio de 2017.}

\section{INTRODUCCION}

La principal función del árbitro de fútbol es aplicar las reglas, para ello debe valerse del proceso de la percepción cuyo objetivo es extraer información del medio a través de los sentidos, para el colegiado el canal de información más importante es el visual que coloca en sus conocimientos estímulos del medio para transformarlos en información. Al enfocar el tema de visual no podemos dejar de lado el campo perceptivo pues se complementan para conformar el proceso de cognición visual que da paso a los procesos de elaboración de la información. Erikson (2007) evidencia acerca de la percepción visual, los movimientos de los ojos y la visión periférica apoya la idea de que una excelente información visual es un fundamento vital para el funcionamiento del mecanismo de percepción, en el modelo de procesamiento de la información visual para un desempeño de calidad. Moreno, Del Campo, Avila y Sabido (2003) expresan su conocimiento definiendo la percepción como un proceso primario en la elaboración de la información, que hace que sea un factor clave en toda acción deportiva. El árbitro es un deportista y como cual debe tener una preparación para lograr su buen desempeño, la preparación no debe estar supeditada solo a la parte física y técnica si no complementarse con una preparación psicológica que le dé la

posibilidad de relacionar y potenciar el proceso visual con la las variables psicológicas y con el campo perceptivo, es acá en donde el juez pone a prueba su nivel atencional la concentración la personalidad y variables especificas del campo perceptivo visual como son el índice de velocidad de procesamiento, que no es otro aspecto si no de poder realizar los procesos mentales para procesar la información visual en el menor tiempo posible puesto que así lo requiere esta actividad del arbitraje, índice de memoria de trabajo que va potenciando en el colegiado la capacidad de almacenar información para sucesos futuros potenciando la experiencia, el índice de razonamiento perceptivo que da la posibilidad de procesar la información percibida por los sentidos y el índice de comprensión verbal que da la posibilidad al árbitro de comprender estímulos verbales que también forman parte de la información adquirida. Todas estas variables psicologías se canalizan en el índice de coeficiente intelectual .El fin de esta investigación es el de crear un modelo para el estudio del campo perceptivo visual en los árbitros de fútbol. En muchas ocasiones nos damos cuenta de que en la toma de decisiones hay acciones no pertinentes algunas por el mismo desarrollo del fútbol y en otras ocasiones por no estar en plena búsqueda de la información en el debido campo. El ser humano en toda actividad que desempeña necesitas adquirir el conocimiento para poder desenvolverse en su respectivo entorno, en el árbitro es el campo de juego acá se busca la información a través de estímulos algunos irrelevantes que se desechan y otros relevantes que se analizan se comparan con la norma y se toma la decisión. Como es de conocimiento en la actualidad el deporte es una de las industrias más poderosas con las que cuenta la humanidad y más especificamente el futbol, puesto que una sociedad que prima el poder económico encontrando acá el espacio para los grandes negocios y por ende todo el entorno del fútbol día a día busca el mejoramiento continuo , los técnicos, los clubes, la parte medica deportiva los medios de comunicación, la infraestructura ,y por lo tanto los árbitros están en la obligación de obtener una gran preparación para estar a la altura del entorno deportivo arbitral. Encontramos procesos que dieron un resultado positivo en la aplicación de juego real dado al buen manejo del modelo del campo de la percepción visual y su relación con las variables psicológicas influenció 
positivamente los resultados. En este aspecto nos encontramos con unos resultados muy positivos en la aplicación de las diferentes fases y se demuestra el desarrollo de relación del campo perceptivo y las variables psicológicas enmarcadas en un buen desempeño del campo de la percepción en la fase de situación real. Concluimos de que el conocimiento el buen manejo del campo de la percepción visual, la preparación psicológica maximiza el rendimiento arbitral al estar alta mente relacionadas las variables psicológicas con el campo perceptivo visual que brinda la posibilidad de tomar decisiones respaldadas por una información extraída por el canal visual dando objetividad al arbitraje.

\section{DESARROLLO METODOLOGICO}

El estudio en mención se llevó a cabo con el colegio de árbitros del Norte de Santander (COARNOS). La muestra está conformada por siete árbitros de sexo masculino, quienes conforman el colegio de árbitros de Norte de Santander que con su conocimiento y desarrollo arbitral atienden los diferentes partidos de fútbol convirtiéndose en fuente de apoyo para poder realizar los diferentes campeonatos.

Tipo de investigación descriptivo transversal de donde la muestra de estudio presenta un buen nivel de conocimiento y experiencia y da confiablidad del proceso y resultados como consecuencia de su alta experiencia en las diferentes categorías del fútbol.

Haciendo un análisis de los componentes de este estudio investigativo se aplicaron tres fases para su desarrollo ver figura 1 :

Primera fase de diagnóstico, en donde se aplicaron a los arbitro objeto de estudio los siguientes procedimientos: examen de agudeza visual, examen de campimetría, los test: mips inventario millon de estilos de personalidad, el WAIS-iv escala wechsler de inteligencia para adultos.

Segunda fase de preparación (operatividad del pensamiento) se aplicaron actividades a la muestra, para el desarrollo de actividades del manejo del campo visual, relacionadas con las variables psicológicas del arbitraje que se relacionan con el campo perceptivo visual.

Tercera fase de situación real, se desarrollaron y grabaron cuatro partidos de fútbol en donde se analizó el desempeño arbitral, para tener conocimiento de cómo el proceso ha influenciado en el rendimiento arbitral.

Modelo ilustrativo del desarrollo metodológico para la aplicación de las diferentes fases del estudio al proceso del estudio del campo de la percepción visual.

Figura 1. Modelo para el estudio del campo de la percepción visual en los árbitros de futbol

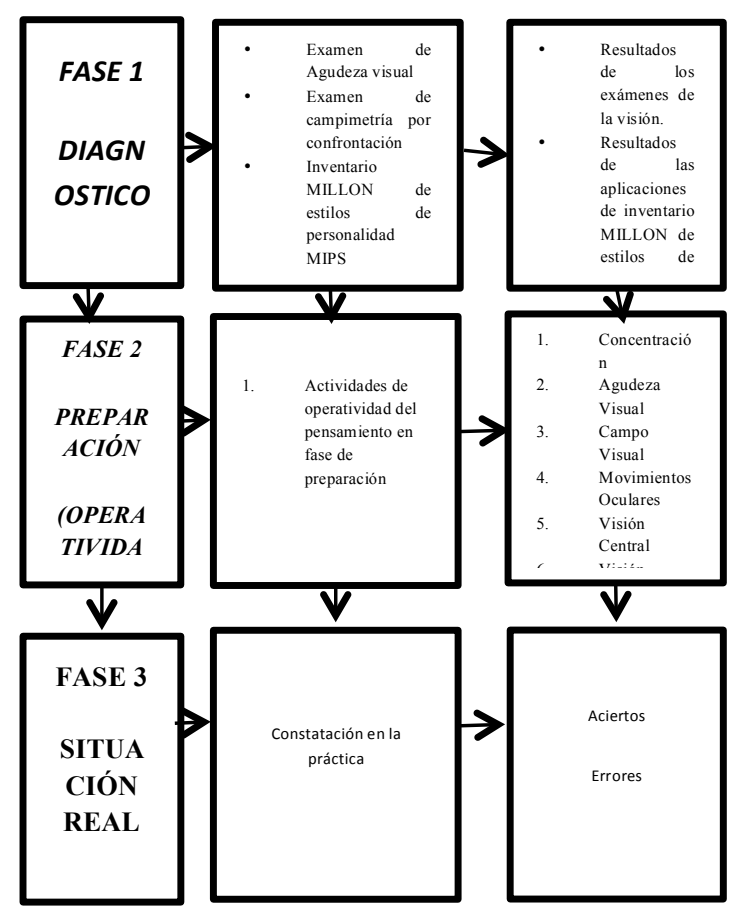

Los instrumentos y metodos nos permite la información directa del desempeño arbitral de los colegiados, la observacion nos ayudo que durante los partidos, daba la opción de registrar periodos de tiempo de 5 minutos las actuaciones arbitrales en los partidos de fútbol. Valoración: Es el indicador utilizado para el estudio del campo de la percepción visual en los árbitros de futbol, con los calificativos de excelente y mala según la decisión tomada en la situación real de juego.

\section{Instrumentos por Fases}

Fase uno diagnóstico:

Examen de agudeza visual el objetivo es conocer el estado de agudeza visual de los árbitros, Lo que busca es detectar cuál es el tamaño de letras más pequeño que una persona puede leer a cierta distancia en una tabla optométrica o en una tarjeta estandarizada, para determinar cuán bien esta su visión. La tabla donde se registran (Snell) en honor al 
especialista que la diseñó en el año 1862. Así, la cantidad de letras que hayas detectado en esta prueba determinará el estado de tu visión. Se considera que a mayor número de líneas identificadas, mayor es tu agudeza visual, es decir que mejor está su vista. Entre las afecciones más conocidas en la agudeza visual, son la miopía (una enfermedad de la vista que hace que los objetos que están cerca se vean con claridad, pero no así los que están lejos), hipermetropía (una condición ocular que hace que los objetos que están lejos se vean bien pero no los que están cerca), astigmatismo (que se produce como resultado de una curvatura irregular del ojo, que afecta el modo en que éste procesa la luz y hace que la visión sea borrosa) o presbicia (una disminución de la capacidad en enfocar con claridad los objetos cercanos, que se produce de manera progresiva como consecuencia natural del envejecimiento). Además, la prueba de agudeza visual permite identificar defectos visuales producidos por otras patologías oculares como cataratas, enfermedades de la retina o del nervio óptico, que deberán ser estudiados con técnicas más específicas.

Examen de campimetría por confrontación tener conocimiento de la capacidad visual de los árbitros, el árbitro permanece sentado frente al examinador. Se examinarán los dos ojos de forma independiente para lo cual el paciente deberá cerrar uno de sus ojos y mirar fijamente al ojo contrario de su examinador mientras se le pide que indique cuando ve y/o cuando deja de ver un objeto (bolígrafo, dedo del examinador...) que se irá desplazando a lo largo del campo visual del ojo examinado. Posteriormente se repetirá la misma maniobra con el ojo contralateral. El estudio del campo visual es una técnica sencilla que puede aportar información muy valiosa al médico no sólo sobre enfermedades que pueden afectar únicamente al globo ocular como el glaucoma, enfermedades de la retina o del nervio óptico; sino también sobre enfermedades locales 0 sistémicas que pueden manifestarse como un defecto del campo visual como enfermedades degenerativas 0 tumorales cerebrales, hipertensión arterial, diabetes y dislipemias, entre otras, cuyo estudio diagnóstico deberá completarse posteriormente con técnicas específicas.

Inventario Millon de estilos de personalidad Autor: Theodore Millon- como todo procedimiento se realizó teniendo en cuenta protocolo y personas especializadas en el tema,tiempo de aplicación aproximadamente 30 minutos-edad de aplicación desde los 18 años hasta los 65-formas de aplicación individualárea que evalúa personalidad- inventario Millón de estilos de personalidad (MIPS) es un cuestionario compuesto por 180 ítems respecto de los cuales el sujeto debe determinar si le son aplicables (respuestas verdadero/falso); su objetivo es medir la personalidad de individuos entre 18 y 65 años o más años, para completar la mayoría de los ítems se requiere un nivel de educación correspondiente al primer año de la escuela secundaria. La mayoría de las personas emplean 30 minutos o menos para responder el cuestionario.

WAIS-IV ESCALA WECHSLER DE INTELIGENCIA PARA ADULTOS.se aplicaron los protocolos a los árbitros de La escala Wechsler de inteligencia para adultos (WAIS-IV) contando con los servicios de personas con experiencia y conocimiento en el tema, es un instrumento clínico de aplicación individual diseñada para evaluar la capacidad cognitiva en adolescentes y adultos con edades de 16:0 a 90:11 años. Esta es una revisión de la escala wechsler de inteligencia para adultos (WAIS-III; Wechsler, 1970) que proporciona puntuaciones de las subpruebas y puntuaciones que representan el funcionamiento intelectual (es decir, $\mathrm{Cl}$ total) Se compone de 15 subpruebas. Doce de ellas fueron conservadas de WAIS III: Diseño con cubos, semejanzas, retención dígitos, matrices,vocabulario, aritmética, búsqueda de símbolos, información, claves, selección de números y letras, comprensión y figuras completas.

Fase dos de preparación Se realizaron y se aplican una serie de actividades relacionadas al campo perceptivo visual que influenciaran el manejo de situaciones que se presentaran en la práctica del árbitro de futbol, que son en la realidad variables del proceso del manejo: concentración, atención, manejo de visión central, aplicación de visión periférica, empleo de campo visual, movimientos oculares .

Fase tres de situación real. Observación de los encuentros. Valiéndonos de cámaras de video de alta calidad y con personal especializado en el oficio, se procedió a realizar grabaciones en las canchas de futbol de noviembre de la ciudad de los patios y lomitas, al desempeño arbitral con el objetivo de observar los árbitros de la investigación y poder constatar en la práctica 
como el desarrollo metodológico ha influenciado en la mejora del arbitraje en cuanto al manejo y aplicación del campo perceptivo visual, en donde mediante análisis se pudo detectar decisiones acertadas en la mayoría de las penalizaciones, se analizaron 4 partidos periodizados en tiempos de 5 minutos en donde la mayoría de intervenciones arbitrales estuvieron de acuerdo al reglamento

\section{RESULTADOS.}

En los aspectos aplicativos de la agudeza visual y campimetría por confrontación se obtuvieron resultados dentro de los rangos normales para ejercer la actividad arbitral, el dictamen del optómetra así lo indica.

\section{PRIMERA FASE}

En primer lugar se obtuvo la matriz de correlaciones, para ver que tanto las variables inmersas dentro de los WAIS-IV, MIPS, CAMPO VISUAL, ACCIONES DE juego reales se relacionan. Posterior a esta se presenta los resultados de la técnica denominada "Análisis de Factores" a fin de reducir variables y buscar unos factores que expliquen mejor la relación entre las variables y en tercer lugar se aplica el análisis de correspondencia simples con el fin de establecer relaciones entre algunas variables de tipo cualitativo partiendo de la tabla de contingencia, la cual verifica si hay 0 no dependencia 0 independencia entre las variables.

En general, se pudo observar que para valorar la personalidad se tiene que evaluar necesariamente la inteligencia. En ambos aspectos se intenta cualificar algo que no se puede ver ni tocar y en ambos casos una valoración debe ser confiable y valida a su vez, al valorar la personalidad lo que se quiere es averiguar la conducta típica del individuo es decir cómo se comporta en las situaciones que convive, al valorar la personalidad implica no solo por conocimiento simple de una persona si no que se tiene que aplicar algunos instrumentos validados como una entrevista, la observación directa del comportamiento aplicado a todas las actividades que se desempeñen, aplicar algunos test que científicamente arrojaran resultados de la personalidad individual, en el arbitraje la valoración de la personalidad es una de las exigencias fundamentales pues es este aspecto una de las variables psicológicas en el desarrollo arbitral.

Su valoración es muy importante para verificar si su personalidad ya que describe los rasgos de personalidad de un individuo, si su personalidad es tan sólida para afrontar retos de muchas responsabilidad y decisión en diferentes situaciones de juego tan exigentes como lo exige un árbitro de fútbol. En las tablas 3 y 4 se dan las escalas de valoración cualitativa y los puntajes de personalidad arrojados por los test MIPS de estilos de personalidad.

Tabla 1. Valoración cualitativa

\begin{tabular}{|l|l|l|l|}
\hline \multicolumn{4}{|c|}{ VALORACIÓN CUALITATIVA } \\
\hline $0-20$ & MUY BAJA & 61 & BUENA(Normal) \\
& & - & \\
& & 80 & \\
\hline $21-40$ & BAJA & 81 & EXCELENTE \\
& & - & \\
& & 10 & \\
& & 0 & \\
\hline $41-60$ & REGULAR & & \\
\hline
\end{tabular}

Tabla 2. Puntajes individuales de personalidad MIPS

\begin{tabular}{|c|c|c|c|c|}
\hline Aspecto & $\begin{array}{l}\text { Arbit } \\
\text { ros }\end{array}$ & $\begin{array}{l}\text { Punt } \\
\text { os }\end{array}$ & $\%$ & $\begin{array}{l}\text { Valoracion } \\
\text { cualitativa }\end{array}$ \\
\hline & 1 & 70 & 70 & Buena (normal) \\
\hline 유 & 2 & 67 & 67 & Buena (normal) \\
\hline 응 & 3 & 60 & 60 & Regular \\
\hline ్ํㅇ & 4 & 75 & 75 & Buena (normal) \\
\hline$\frac{5}{\alpha}$ & 5 & 64 & 64 & Buena (normal) \\
\hline ฉ & 6 & 68 & 68 & Buena (normal) \\
\hline & 7 & 82 & 82 & Excelente \\
\hline
\end{tabular}

Como se puede apreciaren la tabla $\mathrm{N} 1$ se observan las valoraciones cualitativas que marcan unos puntajes según el estudio, estos para ubicarlos en un respectivo rango de acuerdo al puntaje obtenido con la aplicación del test. En la tabla $\mathrm{N} 2$ se observa la valoración cualitativa y nos indican diferentes valoraciones obtenidas por los árbitros de fútbol según su puntuación caracterizan a personalidades buenas o dentro de rango "normal" para ejercer las funciones de árbitro 


\section{Resultados del WAIS IV}

Figura 2. Valoraciones del WAIS IV en los árbitros de fútbol

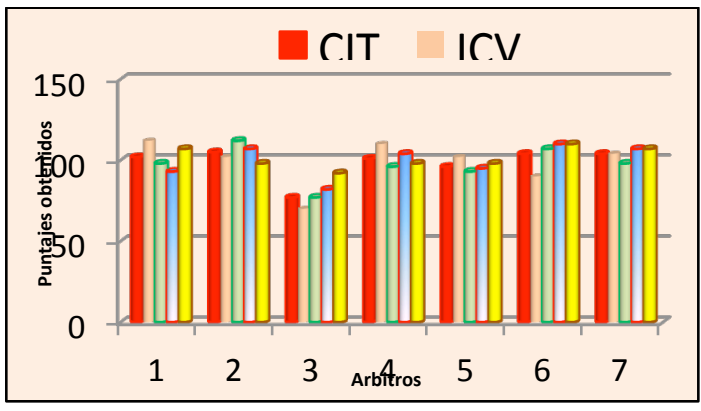

Como podemos observar en la gráfica 2, las valoraciones de la aplicación del WAIS IV hay una alta correlación entre el cociente intelectual total, con el índice de comprensión verbal, índice de razonamiento perceptivo, índice de memoria de trabajo e índice de velocidad de procesamiento, como lo indica la figura uno los resultados son muy comunes y aptos en los árbitros objeto del estudio.

Tabla 3. Medidas de la aplicación del WAIS IV en los árbitros

\begin{tabular}{|l|l|l|l|l|l|}
\hline $\begin{array}{l}\text { Sujeto } \\
\mathrm{s}\end{array}$ & Cit & Icv & Irp & Imt & Ivp \\
\hline 1 & 102 & 112 & 98 & 93 & 107 \\
\hline 2 & 105 & 102 & 112 & 107 & 98 \\
\hline 3 & 77 & 70 & 77 & 82 & 92 \\
\hline 4 & 101 & 110 & 96 & 104 & 98 \\
\hline 5 & 96 & 102 & 93 & 95 & 98 \\
\hline 6 & 104 & 90 & 107 & 110 & 110 \\
\hline 7 & 104 & 104 & 98 & 107 & 107 \\
\hline media & $\begin{array}{l}98,4285 \\
714\end{array}$ & $\begin{array}{l}98,5714 \\
286\end{array}$ & $\begin{array}{l}97,2857 \\
143\end{array}$ & $\begin{array}{l}99,7142 \\
857\end{array}$ & $\begin{array}{l}101,428 \\
571\end{array}$ \\
\hline $\begin{array}{l}\text { d. } \\
\text { Están } \\
\text { dar }\end{array}$ & $\begin{array}{l}9,91391 \\
518\end{array}$ & $\begin{array}{l}14,4551 \\
853\end{array}$ & $\begin{array}{l}11,1312 \\
516\end{array}$ & $\begin{array}{l}10,0947 \\
888\end{array}$ & $\begin{array}{l}6,57918 \\
797\end{array}$ \\
\hline
\end{tabular}

En la tabla $\mathrm{N} 3$ podemos analizar las medidas cuantitativas de la aplicación del WAIS IV con una media y desviación estándar con correlaciones entre cada una de ellas, lo que nos indica la aplicabilidad de estas variables psicológicas en los árbitros.

Luego de hacer un análisis de los factores se detecta que la agudeza visual, el campo visual ,las variables psicológicas determinadas por la aplicación de los test realizados en la primera fase son determinantes en el desarrollo informativo adquirido a través del campo perceptivo y de recopilar la información aplicando la técnica de análisis de correspondencia para buscar comunalidades entre las diferentes variables mediante la correlación entre ellas.se determino que la aplicación de los test MIPS-WAIS IV, son determinantes para detectar las variables psicológicas correlacionadas con el campo perceptivo.

\section{FASE DOS}

Esta fase nos da a conocer la importancia de la operatividad del pensamiento en situación de preparación, en los árbitros. Se desarrolló la intervención de la aplicación de actividades tabla 4 con el objetivo de lograr un perfeccionamiento en donde se desarrollan habilidades psíquicas, atencionales, de concentración, de agilidad mental, de coordinación ocular, manejo de campo visual, aplicación de movimientos oculares, ejercicios de visión central, periférica y el desarrollo del mecanismo relacionado con el campo perceptivo indicado por los índices del WAIS IV .

Tabla 4. Plan de trabajo de intervención, Actividades del campo perceptivo visual

\begin{tabular}{|c|c|c|c|c|c|c|c|c|c|c|c|c|}
\hline \multirow{2}{*}{$\frac{\text { Meses }}{\text { Semanas }}$} & \multicolumn{4}{|c|}{ Febrero } & \multicolumn{4}{|c|}{ Marzo } & \multicolumn{4}{|c|}{ Abril } \\
\hline & 1 & 2 & 3 & 4 & 5 & 6 & 7 & 8 & 9 & $\begin{array}{l}1 \\
0\end{array}$ & $\begin{array}{l}1 \\
1\end{array}$ & $\begin{array}{l}1 \\
2 \\
\end{array}$ \\
\hline Días & 2 & 2 & 2 & 2 & 2 & 2 & 2 & 2 & 2 & 2 & 2 & 2 \\
\hline Tiempo & $\begin{array}{l}6 \\
0\end{array}$ & $\begin{array}{l}6 \\
0 \\
,\end{array}$ & $\begin{array}{l}6 \\
0 \\
,\end{array}$ & 0 & $\begin{array}{l}6 \\
0 \\
,\end{array}$ & $\begin{array}{l}6 \\
0 \\
,\end{array}$ & $\begin{array}{l}6 \\
0 \\
,\end{array}$ & $\begin{array}{l}6 \\
0 \\
,\end{array}$ & $\begin{array}{l}6 \\
0 \\
,\end{array}$ & $\begin{array}{l}6 \\
0 \\
,\end{array}$ & $\begin{array}{l}6 \\
0\end{array}$ & $\begin{array}{l}6 \\
0 \\
,\end{array}$ \\
\hline \multirow{3}{*}{$\begin{array}{l}\text { Actividades del } \\
\text { campo } \\
\text { perceptivo } \\
\text { visual }\end{array}$} & \multicolumn{4}{|c|}{$\begin{array}{l}\text { Volumen de } \\
\text { tiempo 480' }\end{array}$} & \multicolumn{4}{|c|}{$\begin{array}{l}\text { Volumen de } \\
\text { tiempo 480' }\end{array}$} & \multicolumn{4}{|c|}{$\begin{array}{l}\text { Volumen de } \\
\text { tiempo 480' }\end{array}$} \\
\hline & \multicolumn{12}{|c|}{ Nombre de las actividades aplicadas } \\
\hline & \multicolumn{4}{|c|}{$\begin{array}{l}\text {-desarrollo de } \\
\text { laberintos } \\
\text { gráficos } \\
\text {-figuras con el } \\
\text { tangram } \\
\text {-estimulación } \\
\text { visual, } \\
\text { cognitiva y } \\
\text { atencional } \\
\text {-sopa de letras } \\
\text { con } \\
\text { localización de } \\
\text { palabras } \\
\text { alusivas al } \\
\text { campo } \\
\text { perceptivo } \\
\text { visual } \\
\text {-ejercicios de } \\
\text { motricidad fina }\end{array}$} & \multicolumn{4}{|c|}{$\begin{array}{l}\text {-figuras con el } \\
\text { cubo soma } \\
\text {-expansión } \\
\text { progresiva } \\
\text {-actividades de } \\
\text { movimientos } \\
\text { oculares } \\
\text {-manipulación } \\
\text { de laberintos } \\
\text { manuales } \\
\text {-juegos de } \\
\text { apilamiento } \\
\text { rápido } \\
\text { deportivo }\end{array}$} & \multicolumn{4}{|c|}{$\begin{array}{l}\text {-dominio visual } \\
\text { entre dos } \\
\text { personas } \\
\text {-concéntrese } \\
\text {-ejercicios de } \\
\text { visión } \\
\text { periférica } \\
\text {-ejercicios con } \\
\text { las torres de } \\
\text { hanói } \\
\text {-buscar } \\
\text { diferencias }\end{array}$} \\
\hline
\end{tabular}




\section{TERCERA FASE}

Se muestran los resultados de situación real de los partidos que arbitran los colegiados, se efectuaron grabaciones en su desempeño y se puedo observar de cómo el proceso metodológico de la investigación ha influido en el buen desarrollo del arbitraje, al ellos tener un mejor manejo del campo de la percepción visual que como lo hemos manifestado con anterioridad es lo que le da la objetividad al árbitro por ser la percepción visual la principal fuente de información del ser humano en toda actividad que desempeñe, como podemos observar en las figuras ocho y nueve los indicadores de acciones de los árbitros informan que hubo un gran acierto en cuanto toma de decisiones para aplicar el desarrollo de los partidos, es muy importante saber por estos indicadores que el proceso ha influenciado en el desempeño arbitral mediante resultados $y$ motivaciones expresadas por los colegiados con el desarrollo metodológico que ha despertado en ellos ese afán de superarse para lograr un buen desempeño mediante preparaciones que integren más los aspectos científicos para alcanzar un desempeño de excelencia arbitral.

Figura 3. Juego real primer tiempo de fecha 14 marzo 2015

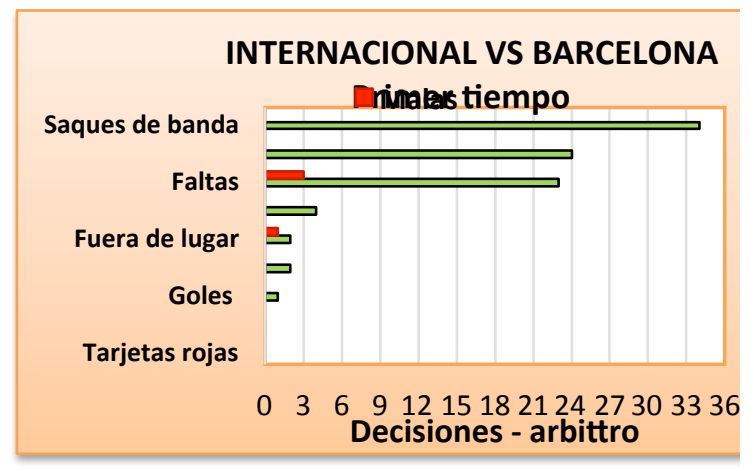

Figura 4. Juego real segundo tiempo de fecha 14 marzo 2015

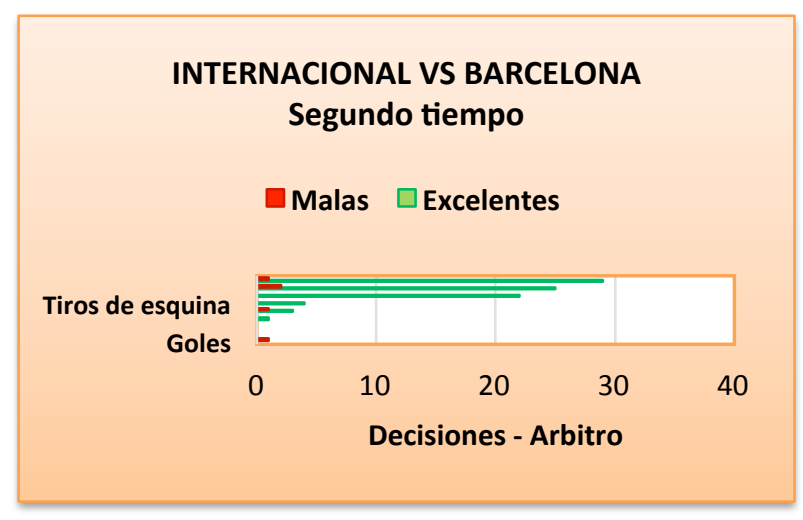

Se observa en las figuras 3 y 4 que en el partido real de 192 decisiones registradas sólo 5 fueron clasificadas como malas. En otras palabras se puede determinar que aproximadamente el $95 \%$ de las decisiones tomadas por el árbitro fueron excelentes.

\section{DISCUSION}

El objetivo principal de esta investigación fue la de crear el modelo anteriormente citado y comparar el mismo frente a la metodología propuesta por Ortega A, Montañez G (2012) aplicado al estudio del campo preceptivo visual en las selecciones deportivas de la Universidad de Pamplona. Después de analizar los tratamiento estadístico de los datos, se observa en los resultados obtenidos en este estudio que con las dos metodologías se consiguen mejoras deportivas en cuanto tiene que ver con el campo perceptivo visual puesto que se encuentran correlaciones muy parecidas con las variables psicológicas y componentes del funcionamiento del campo perceptivo visual, en la investigación del modelo del para el estudio del campo de la percepción visual en los árbitros profesionales de Norte de Santander se observan correlaciones más numerosas puesto que esta investigación está más compleja por estar direccionada a un aspecto de dimensiones extensas por indicar el manejo visual específicamente correlacionado con aspectos del funcionamiento psíquico dado por las variables psicológicas, en el artículo de Ortega, Montañez, el estudio visual es más sesgado por ir enfocado a deportistas que no deben ejercer una visión tan constante como los árbitros.

\section{CONCLUSIONES}

Al observar los resultados del desempeño arbitral en situación real se determina un alto 
grado de efectividad del $90 \%$ en los árbitros influenciado por el buen manejo del campo del a percepción visual.

El campo perceptivo visual tiene que estar complementado con las variables psicológicas, atención, concentración, índice de coeficiente intelectual, índice de capacidad verbal, índice perceptivo, índice de memoria de trabajo, índice de velocidad de procesamiento para lograr el buen desempeño arbitral con porcentajes equitativos dado que las situaciones de juego requiere una aplicación psicológica total, para lograr el buen desempeño arbitral.

La información visual es la principal fuente de información del árbitro de fútbol por permitir extraer información del medio realizar los procesos mentales desechar las información irrelevante analizar la información relevante y aplicar la norma, la percepción visual aporta el $85 \%$ de la información al árbitro y se complementa con la percepción auditiva. Esto da la posibilidad de un arbitraje objetivo.

\section{REFERENCIAS BIBLIOGRAFICAS}

González, I. Casáis, L. (2011) Comparación de la atención visual y campo visual en deportistas en función de nivel de pericia .Universidad de Vigo España .Revista internacional de ciencias del deporte .Editorial: RICYDI. ISSN: 18853137

Maldonado, V. García, L .Contreras, J. (2012) La investigación del comportamiento visual, desde el enfoque perceptivo -cognitivo y la toma de decisiones en el deporte. Castilla España .Editorial:Martos.ISSN: 19896239

Ortega, J. Montañez, G.( 2012 ) Estudios del campo perceptivo visual en las selecciones deportivas de La universidad de Pamplona. Pamplona Colombia. Revista de actividad física y desarrollo humano. Editorial: Upa.

Moreno, F.Avila, F. Damas, S. (2001) El papel de la motilidad ocular extintica en el deporte. Aplicación en los deportes abiertos. Extremadura España. Revista motricidad. Editorial: INDEX. ISSN: 02140071

Palmi, J. (2010) La percepción enfoque funcional de la visión. Madrid España. Revista entrenamiento visual. Editorial: INEFC
Pérez, T. (2011) Ejercicios para el estudio de la percepción visual. Alicante España. Editorial: Club universitario

Quevedo, L.Sole, F. (2007) La visión en el baloncesto .Barcelona España .Archivos de medicina del deporte. Revista internacional de ciencias del deporte

Ramírez, A. Alonso, A. Francesc, F. López, M. (2006) Programa de intervención psicológica con árbitros de fútbol. Barcelona España. Revista de psicología del deporte. Editorial: TEAM. ISSN: 19885636.

Richard, H. (2009) Sensación y percepción un enfoque integrador. Bogotá Colombia. Editorial: manual moderno.

Rivero, M. Conde, M. Fernández, J. (2007) Manual de psicología general, atención y percepción. Madrid España. Editorial Sáenz y Torres

Ruiz, A. Reina, R. Del campo, Sabido, R. Moreno. (2004) Estrategias de búsqueda visuales elaboradas por árbitros de baloncesto c0on diferente nivel de experiencia. Un estudio de casos. Murcia España. Facultad de ciencias del deporte. Universidad de Extremadura.

Ruiz, J. (2010) Árbitro de fútbol: Arbitraje y juicio deportivo. Madrid España. Editorial: DYKYNSON.

Sáez, N.Vila, S. Abellan, H.Contreras, O. (2012) Análisis del comportamiento visual y la toma de decisiones en el bloqueo en voleibol. Murcia España. Cuadernos de psicología del deporte.ISSN:15788423

Sardinero, A. (2011) Taller ejercicios percepción: setenta fichas ejercicios prácticos con soluciones. Madrid España. Editorial: Gesfomedia

Styles, E. (2010) Psicología de la atención. Madrid España. Editorial: UNIVERSITATIA Ramón Areces

Tamorri, S (2009) Neuro ciencia y deporte. Buenos Aires Argentina. Editorial: Paidotribo.

Vargaz, L. (1994) Sobre el concepto de percepción .Iztapalapa México, red de revistas científicas de américa latina y el caribe, España y Portugal. Sistema de información científica.ISSN: 01887017 
Vidal, M. (2011) Taller de menoría: Como organizar un taller para mantener la mente en forma. Barcelona España. Editorial: Alba

Wechsler, D. (2012) Manual de aplicación. Escala Wechsler de inteligencia para adultos WAIS IV, facultad de psicología universidad Nacional Autónoma de Mexico.Editorial: Manual moderno.ISBN:9786074483550 